\title{
IMPLICATIONS OF GLOBAL AND LOCAL MOBILITY IN AMORPHOUS EXCIPIENTS AS DETERMINED BY DSC AND TM DSC
}

\author{
Ion Dranca* and Tudor Lupascu \\ Institute of Chemistry of the Academy of Sciences of Moldova \\ 3 Academiei Str., Chisinau, MD 2028 \\ Email:drancai@yahoo.com
}

\begin{abstract}
The paper explores the use of differential scanning calorimetry (DSC) and temperature modulated differential scanning calorimetry (TM DSC) to study $\alpha$ - and $\beta$ - processes in amorphous sucrose and trehalose. Amorphous sucrose was prepared by lyophilization while amorphous trehalose was prepared by dehydration of trehalose dihydrate. The variation in the effective activation energy of $\alpha$-relaxation through glass transition has been determined by applying an isoconversional method. $\beta$-relaxations were studied by annealing glassy samples at different temperatures and subsequently heating at different rates in a differential scanning calorimeter. The effect of heating rate on the $\beta$-relaxation peak temperature formed the basis for the calculation of the activation energy. The higher density of glassy trehalose resulted in larger activation energy of $\alpha$-relaxation compared to sucrose. The effect of temperature on viscous flow was greater in trehalose which can have implications on lyophile collapse. The size of the cooperatively rearranging regions was about the same for sucrose and trehalose suggesting similar dynamic heterogeneity at their respective glass transition temperatures. The activation energy of $\beta$-relaxations increased with annealing temperature due to increasing cooperative motions and the increase was larger in sucrose. The temperature at which $\beta$-relaxation was detected for a given annealing time was much less in sucrose implying that progression of local motions to cooperative motions occurred at lower temperatures in sucrose.
\end{abstract}

Keywords: molecular mobility; excipients (sucrose \& trehalose); $\alpha$ - and $\beta$-relaxations; activation energy; DSC \& TM DSC.

\section{Introduction}

A number of active pharmaceutical ingredients in the drug development pipelines, are prepared in the amorphous state, in light of their poor aqueous solubility [1]. In addition, many pharmaceutical excipients (an excipient is an inactive substance used as a carrier for the active ingredients of a medication) exist in the amorphous state, or are rendered amorphous in order to obtain the desired functionality [2]. Since amorphous compounds are inherently less stable, both physically and chemically, than their crystalline counterparts, numerous investigations over the last decade expectedly focused on the stabilization of amorphous pharmaceuticals [3-5].

A number of protein pharmaceuticals are prepared by lyophilization, and the stresses encountered during lyophilization can cause destabilization of the protein. Lyoprotectants are excipients (typically disaccharides) which help in the preservation of the native state of proteins either by reducing the conformational flexibility of proteins by being in the highly viscous glassy state or by hydrogen bonding [2]. Crystallization of these lyoprotectants can result in protein destabilization [6-10]. Izutsu et al.[8] reported that devitrification of inositol resulted in the instability of a model protein and addition of water soluble polymers stabilized the protein by preventing inositol crystallization. Sucrose and trehalose are widely used as lyoprotectants. Liao et al.[11] reported sucrose to be more effective than trehalose in stabilizing spray-dried lysozyme. On the other hand, Sun et al.[12] compared the effectiveness of sucrose and trehalose to stabilize protein formulations and attributed the superiority of trehalose to its lower free volume in the glassy state as well as lower free volume expansion coefficient above the glass transition temperature $\left(T_{g}\right)$. Both these properties indicated more restricted molecular motions in amorphous trehalose than in amorphous sucrose, both in the glassy in the rubbery states. The relative effectiveness of maltitol, sucrose and trehalose, three model compounds, depends all the vitrification mechanism and specific interactions in a particular formulation.

Molecular mobility in glassy pharmaceuticals has been studied by differential scanning calorimetry and temperature modulated DSC with the goal of generating information that would aid in the prediction of physical stability. Most of these reports [13-16] deal with the molecular motion associated with glass transition or the cooperative $\alpha$-relaxation. Information on the mobility below $\mathrm{T}_{\mathrm{g}}$, derived by extrapolation from the data obtained above $T_{g}$, is at best an indirect measure of the local mobility or the non-cooperative $\beta$-relaxation. These secondary intermolecular relaxations were present in all small molecule organic glasses and are referred to as the JohariGoldstein relaxations [17,18]. In contrast to $\alpha$-relaxations, they generally follow Arrhenius kinetics and are characterized by activation energy values that are much smaller than those of $\alpha$-relaxations.

The $\beta$-relaxations, specifically the Johari-Goldstein relaxations, are supposed to be the precursor to the cooperative $\alpha$-relaxations[19]. Thus their characterization is immensely important both for understanding the nature of molecular motions in the glassy phase and their role in determining physical stability. Hikima et al. concluded that $\beta$-relaxation processes control the crystal growth in triphenylethylene [20]. Similar observations were made by Alie 
et al. [21] who showed that $\beta$-relaxations can control the process of crystal growth of a low molecular weight compound above $\mathrm{T}_{\mathrm{g}}$. Although dielectric spectroscopy has been typically used to study structural relaxations, recently Vyazovkin et al. have shown that it is possible to study both $\alpha$ - and $\beta$-relaxations using conventional DSC $[22,23]$. While relaxations far below $T_{g}$ are non-cooperative (i.e. $\beta$ ), cooperative $\alpha$-relaxations evolve as $T_{g}$ is approached and cause glass transition. By annealing amorphous systems below $\mathrm{T}_{\mathrm{g}}$, followed by rapid cooling and reheating, $\beta$-relaxation is manifested by a shallow enthalpic recovery endotherm preceding the $T_{g}$. With an increase in annealing time, the enthalpic recovery peak shifts to higher temperatures. This effect was systematically investigated in inorganic glasses [24]. Bershtein and Egorov [25] studied the same effect in polymers and used the relationship between the peak temperature, $\mathrm{T}_{\mathrm{p}}$, and the heating rate, $\mathrm{q}$, to calculate activation energy, $\mathrm{E}$ :

$$
E=-R \frac{d \ln q}{d T_{p}^{-1}}
$$

Our overall goal is to gain a mechanistic understanding of the differences in the global (glass transition) and local mobility in amorphous sucrose and trehalose In light of the differences in their properties, it will be worthwhile to comprehend the implications of molecular mobility on their role as lyoprotectants. Our first objective is to demonstrate the utility of a macroscopic property, specifically density, as a predictor of the temperature dependence of global mobility. Although the densities of crystalline sucrose and trehalose (anhydrous) are almost the same $\left(\sim 1.58 \mathrm{~g} \mathrm{~cm}^{-3}\right)$ [4], the density of glassy trehalose $\left(1.54 \mathrm{~g} \mathrm{~cm}^{-3}\right)$ is higher (lower free volume) than that of glassy sucrose $\left(1.43 \mathrm{~g} \mathrm{~cm}^{-3}\right)$ [26]. This can be attributed to the nature of hydrogen bonding network present in trehalose (discussed in detail later). Thus we anticipate higher activation energy values through the glass transition for amorphous trehalose compared to amorphous sucrose. Our second objective is based on the hypothesis that the difference in activation energy for global mobility between the glassy and supercooled liquid states would be more for trehalose than for sucrose. In other words, the effect of temperature in reducing the activation energy barrier for the glass to flow would be more in trehalose than in sucrose. Our third objective is to measure the minimum temperature of $\beta$-relaxation in amorphous sucrose and trehalose under similar experimental conditions (e.g., annealing time). Assuming that $\beta$-relaxations are the precursor to the glass transition or the global mobility, we hypothesize that the minimum temperature of $\beta$-relaxation would be more in trehalose compared to sucrose because amorphous trehalose has a higher $\mathrm{T}_{\mathrm{g}}$ than sucrose. Lastly, as an indirect proof of the thermally induced progression of local to global mobility, we aim to show that the activation energy of $\beta$-relaxation approaches that of the $\alpha$ relaxation as the annealing temperature approaches $\mathrm{T}_{\mathrm{g}}$

\section{Materials and Methods}

Crystalline sucrose and trehalose dihydrate (Sigma, St. Louis, MO, USA) were used as obtained.

\section{Differential scanning calorimetry (DSC)}

A differential scanning calorimeter (MDSC 2920, TA instruments, New Castle, DE and Mettler-Toledo DSC 823) equipped with a refrigerated cooling accessory was used. The instrument was calibrated with pure indium and tin. About 15-20 mg of sample was weighed in an aluminum pan which was then crimped non-hermetically.

For sub- $\mathrm{T}_{\mathrm{g}}$ measurements ( $\beta$-relaxation), samples were quench cooled in liquid nitrogen and immediately placed in the DSC cell (Mettler-Toledo DSC 823 and TA Instruments MDSC 2920) maintained at $-40^{\circ} \mathrm{C}$. The samples were then heated to the annealing temperature and held for 30 minutes. The annealing temperatures were -25 , $-20,-10,-5,0,5,10{ }^{\circ} \mathrm{C}$ for sucrose and $-10,0,10,20,30,35,40,50,60,70^{\circ} \mathrm{C}$ for trehalose. After completion of the annealing segment, the samples were cooled down to $-40^{\circ} \mathrm{C}$ and immediately heated above the $\mathrm{T}_{\mathrm{g}}$. The heating rates were $10,15,20,25$ and $30^{\circ} \mathrm{C} \mathrm{min}^{-1}$. The resulting endothermic peaks detected upon heating were used to determine the peak temperature by using the standard DSC analysis software (Universal analysis 2000, TA Instruments or Mettler-Toledo, STAR ${ }^{\mathrm{e}}$ 9.01). Prior to these experiments, samples of sucrose and trehalose were heated to $85^{\circ} \mathrm{C}$ and $155^{\circ} \mathrm{C}$ respectively and held at the respective temperatures for 5 minutes to erase thermal history.

For the measurements of glass transition or $\alpha$-relaxation, glassy sucrose and trehalose were heated above their respective glass transition temperatures to erase thermal history. The samples were then cooled to $\sim 40^{\circ} \mathrm{C}$ below their respective glass transition temperatures at $10,15,20,20,25$ and $30^{\circ} \mathrm{C} \mathrm{min}$. The samples were immediately heated at the same rate as that used for cooling. The cell constant was determined and the temperature calibration of the DSC was performed using an indium standard at a heating rate of $20^{\circ} \mathrm{C} \mathrm{min}{ }^{-1}$. The validity of the temperature calibration was checked at all the other heating rates used. The heat capacity of sucrose and trehalose was measured using sapphire as the reference. A Mettler-Toledo DSC823e with an HSS7 sensor was employed for performing DSC measurements that were carried out under nitrogen flow $\left(50 \mathrm{ml} \cdot \mathrm{min}^{-1}\right)$. Heat and temperature calibrations were performed by using gallium and indium standards. Regular DSC runs were conducted by heating the samples from 25 to $90{ }^{\circ} \mathrm{C}$ at $0.5-5 \mathrm{~K} \cdot \mathrm{min}^{-1}$. The regular DSC was complemented by the recently invented (J. E. K. Schawe, T. 
Hutter, C. Heitz, I. Alig, D. Lellinger, Thermochim.Acta 2006, 446, 147) technique of multi-frequency temperature modulated DSC (TOPEM ${ }^{\circledR}$ by Mettler-Toledo). The program temperature for TOPEM ${ }^{\circledR}$ measurements was defined by superimposing the underlying heating rate of $1 \mathrm{~K} \cdot \mathrm{min}^{-1}$ and a series of stochastic temperature pulses of the $1 \mathrm{~K}$ amplitude and the time between the pulses ranging from $25 \mathrm{~s}$ to $60 \mathrm{~s}$. All measurements were performed at least two times to ensure reproducibility.

An example of a multifrequency DSC measurement for sucrose is given in Figure 1. The real part of the complex heat capacity is evaluated at the frequencies, $f$, from 5 to $25 \mathrm{mHz}$.

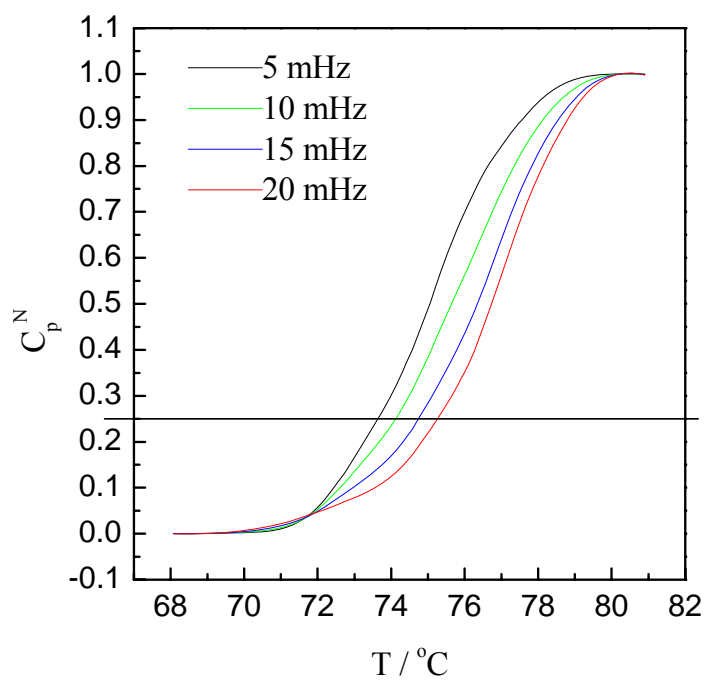

Fig. 1. Frequency dependence of the normalized heat capacity for sucrose evaluated by multifrequency DSC (TM DSC).

\section{Results and Discussions}

Variation of activation energy through the glass transition in amorphous sucrose and trehalose

As mentioned earlier, an advanced isoconversional method was used for this purpose. While we provide the highlights here, the details can be found elsewhere [27-29]. Isoconversional methods are used to describe the kinetics of a complex multi-step process by the application of the Arrhenius model to several single-step components or extents of conversion of the process. These methods can be used to evaluate the variation of activation energy, $\mathrm{E}_{\alpha}$, with the extent of conversion, $\alpha$, by using different heating rates. Vyazovkin [29] proposed an advanced method which involves carrying out a set of $n$ experiments using different temperature programs, $T_{i}(t)$, resulting in the determination of a value of $E_{\alpha}$ that minimizes the following function:

$$
\Phi\left(E_{\alpha}\right)=\sum_{i=1}^{n} \sum_{j \neq 1}^{n} \frac{J\left[E_{\alpha}, T_{i}\left(t_{\alpha}\right)\right]}{J\left[E_{\alpha}, T_{j}\left(t_{\alpha}\right)\right]}
$$

$$
\text { where } \quad J\left[E_{\alpha}, T_{i}\left(t_{\alpha}\right) \equiv \int_{t_{\alpha}-\Delta \alpha}^{t_{\alpha}} \exp \left[\frac{-E_{\alpha}}{R T_{i}(t)}\right] d t\right.
$$

The advantages of this method over other isoconversional methods $[30,31]$ are that it uses the actual sample temperature in order to account for the deviation from linear heating or cooling due to self-heating or cooling. Integration over small temperature segments also results in the elimination of error associated with the variation of $\mathrm{E}_{\alpha}$ with $\alpha$.

The extent of conversion $\alpha$ is determined from the normalized heat capacity, $C_{p}^{N}$, which is evaluated as follows:

$$
C_{p}^{N}=\frac{\left(C_{p}-C_{p g}\right) \mid}{\left.\left(C_{p l}-C_{p g}\right)\right|_{T}} \equiv \alpha
$$

where $\mathrm{C}_{\mathrm{p}}$ is any given heat capacity and $C_{\mathrm{pg}}$ and $\mathrm{C}_{\mathrm{pl}}$ are the heat capacities of the glass and the liquid respectively. 
In Figure 2, the DSC glass transition events (heating rate: $10^{\circ} \mathrm{C} \mathrm{min}^{-1}$ ) in amorphous sucrose and trehalose are shown. The glass transition temperatures (midpoint) of sucrose and trehalose were estimated to be 73 and $119^{\circ} \mathrm{C}$ respectively. The dependence of the activation energy on the extent of conversion $\alpha$ has been determined by applying the advanced isoconversional method outlined above to the $C_{p}^{N}$ (or $\alpha$ ) vs temperature data. Figure 2 shows the change in the activation energy as a function of the extent of conversion for sucrose and trehalose. The decrease in activation energy has been plotted against temperature in Figure 3 by replacing $\alpha$ with the corresponding average temperature at different heating rates. The activation energy decreases as the glass transition event progressed. For trehalose the activation energy for $\alpha$-relaxation decreased from $\sim 348$ to $\sim 312 \mathrm{~kJ} \mathrm{~mol}^{-1}$ whereas for sucrose it changed from $\sim 227$ to $\sim 198 \mathrm{~kJ} \mathrm{~mol}^{-1}$. In the glassy phase the low free volume results in large activation energy for molecular motions. As the temperature is increased, the free volume increases, thereby facilitating molecular motion and lowering the activation energy [32].

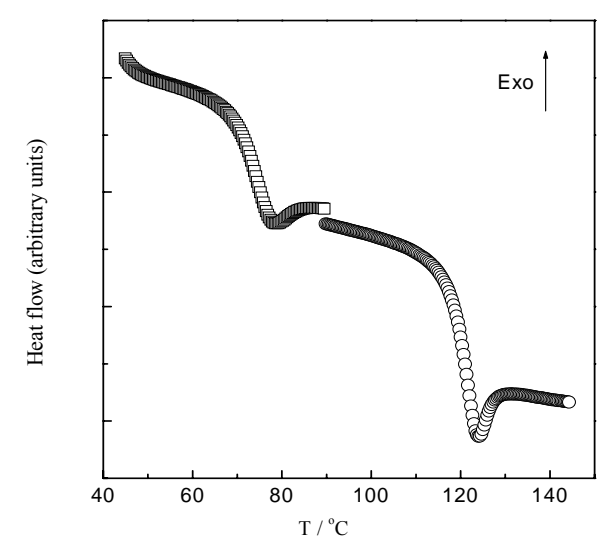

Fig. 2. DSC curves for the glass transition of sucrose (squares) and trehalose (circles) obtained at $10^{\circ} \mathrm{C} \mathrm{min}^{-1}$

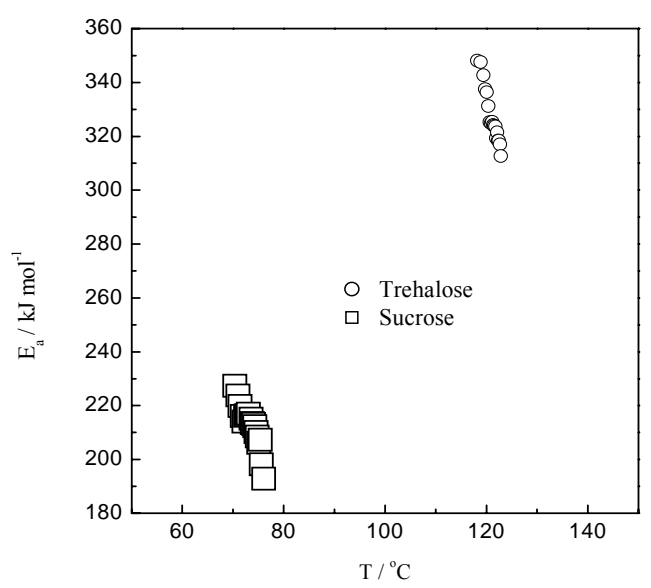

Fig. 4. Variation in the effective activation energy with temperature from the glassy to the liquid state in sucrose (squares) and trehalose (circles).

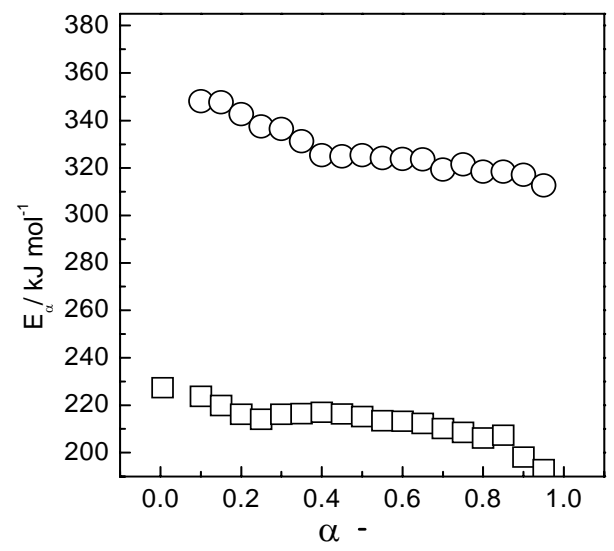

Fig. 3. Variation in the effective activation energy with the extent of conversion from the glassy to the liquid state in sucrose (squares) and trehalose (circles).

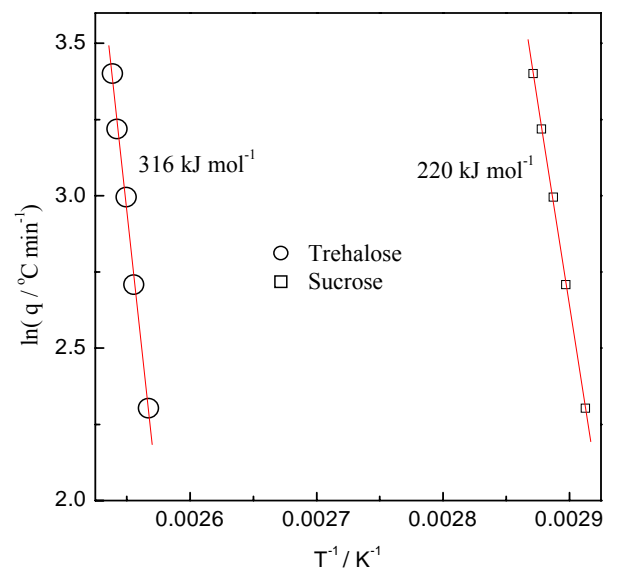

Fig. 5. Evaluation of activation energy (equation 1) of the glass transition in trehalose and sucrose.

The range of activation energy values for trehalose is greater than that for sucrose. Single activation energies for $\alpha$-relaxation in sucrose $\left(220 \mathrm{~kJ} \mathrm{~mol}^{-1}\right)$ and trehalose $\left(316 \mathrm{~kJ} \mathrm{~mol}^{-1}\right)$ were also calculated using Equation 1 and have been shown in Figure 5. Literature reports for activation energy values for trehalose range from 229 to $732 \mathrm{~kJ}$ $\mathrm{mol}^{-1}[33,34]$ whereas those for sucrose range from 152 to $407 \mathrm{~kJ} \mathrm{~mol}^{-1}[33,35]$. Despite the range of activation energy values reported, it is clear that amorphous trehalose is characterized by higher activation energy values of $\alpha$ relaxation compared to sucrose. 
Hancock et al. [33] reported similar findings for amorphous sucrose and trehalose based on the activation energy values calculated from the onset, mid-point and offset $T_{g}$ values. We can therefore conclude that amorphous trehalose has lower free volume and hence more restricted molecular mobility compared to that of sucrose. Sun et al.[12] had earlier reached the same conclusion using the Williams-Landel-Ferry model. The lower free volume of trehalose is probably also reflected in the fact that although both crystalline sucrose and (anhydrous) trehalose have almost the same density $\left(1.58 \mathrm{~kg} \mathrm{~m}^{-3}\right)$, the density of amorphous trehalose $\left(1.49 \mathrm{~kg} \mathrm{~m}^{-3}\right)$ is higher than that of amorphous sucrose $\left(1.43 \mathrm{~kg} \mathrm{~m}^{-3}\right)$ [4]. The higher density of amorphous trehalose could be explained from the hydrogen bonding patterns in the glassy state. Trehalose, composed of two D-glucose rings is bisymmetric compared to sucrose which is characterized by the asymmetry of the constituent glucose and fructose rings. This binary symmetry in the glucose units led Phillips[36] to propose a "tandem sandwich" structure for trehalose. In this structure, strongly bound pairs of glucose rings are alternated with weakly bound pairs resulting in a stable and flexible arrangement. The ethanol side groups of the pyranose rings reside between the rings for weakly bound pairs whereas they lie outside the rings for the strongly bound pairs. Such "sandwich" structures would naturally lead to better packing and hence the higher density of glassy trehalose compared to glassy sucrose where such arrangement is not feasible due to ring asymmetry.

Vyazovkin et al.[32] proposed a variability parameter, $\Delta \mathrm{E}$, to describe the rate of decrease of the activation energy with the average temperature:

$$
\Delta E=\frac{E_{0.25}-E_{0.75}}{T_{0.25}-T_{0.75}}
$$

where $E_{0.25}$ and $E_{0.75}$ are the activation energy values for $\alpha=0.25$ and 0.75 respectively and $T_{0.25}$ and $T_{0.75}$ are the corresponding temperatures. $\Delta E$ could be used as a measure of kinetic fragility or deviation from the Arrhenius temperature dependence. These authors showed that the $\Delta E$ values of various compounds correlated well with the respective values of fragility index. $\Delta E$ values of sucrose and trehalose were calculated to be -2.3 and -7.2 respectively. Thus although, as mentioned earlier, trehalose is characterized by higher activation energy and hence lower free volume compared to sucrose, the decrease in activation energy per unit of temperature throughout the glass transition is higher for trehalose. This could be explained in light of the fact that sucrose and trehalose liquids have very similar fragility values as detailed below. On the other hand, glassy sucrose and trehalose are characterized by different densities reflecting different free volumes. Since glassy trehalose is more tightly packed compared to glassy sucrose, it is clear that the activation energy barrier for global mobility, required for the glass to start flowing, would be initially much higher in trehalose as was shown above. But since both the liquids are similar in terms of fragility, the effect of increasing the temperature could be expected to have a much greater bearing on the free volume in trehalose. In other words we would expect a greater decrease in activation energy per unit temperature through the glass transition which is exactly what we observed.

The "strength parameter" or the D-value obtained from Vogel-Tammann-Fulcher (VTF) equation could be used as an estimate of kinetic fragility index. Literature VTF D-values for sucrose (7.3) and trehalose (5.1) are similar [13]. Since the kinetic and thermodynamic fragility indices generally correlate well [37], we calculated thermodynamic fragility index using the empirical equation proposed by Wang and Angell [38] :

$$
m=56 \frac{T_{g} * \Delta C_{p}\left(T_{g}\right)}{\Delta H_{m}}
$$

where we have used experimentally obtained mid-point $\mathrm{T}_{\mathrm{g}}$ values of $346 \mathrm{~K}$ and $392 \mathrm{~K}$ respectively for sucrose and trehalose and $\Delta C_{p}\left(T_{g}\right)$ value of $0.55 J^{-1} \mathrm{~g}^{-1}\left(188.3 \mathrm{JK}^{-1} \mathrm{~mol}^{-1}\right)$ for both sucrose and trehalose. The heat of fusion, $\Delta H_{\mathrm{m}}$, for sucrose and anhydrous trehalose were found to be $41.4 \mathrm{~kJ} \mathrm{~mol}^{-1}$ [39] and $53.4 \mathrm{~kJ} \mathrm{~mol}^{-1}$ [40] respectively. Using these values, $\mathrm{m}$ was found to be $\sim 88$ for sucrose and $\sim 77$ for trehalose. Thus although the calculated fragility indices for sucrose and trehalose are in agreement with literature values, the $\Delta E$ values calculated above indicate a difference in the temperature dependance of activation energy and hence the change in free volume with temperature. The above observation implies that the effect of temperature on the viscous flow of trehalose during glass transition is much more than that of sucrose. This could have implications in bringing about collapse of a lyophilized cake in that a small increase in temperature around the $T_{\mathrm{g}}$ ' of a formulation containing trehalose would cause a greater viscous flow than that containing sucrose.

\section{Calculation of the size of cooperatively rearranging regions}

The molecular kinetic theory of Adam and Gibbs [41] describes the temperature dependence of structural relaxation in the supercooled liquid phase in terms of the temperature dependence of the size of the "cooperatively 
rearranging regions (CRR) ". CRR is the smallest region that can undergo a change in configuration independent of the neighboring molecules in response to an adequate thermal fluctuation. Donth has united the concept of "islands of mobility" proposed by Johari [17] with the defect diffusion model of Glarum [42]. Molecular mobility is facilitated when a "defect" or a locally concentrated high free volume (island of mobility) diffuses due to thermal fluctuations. These defects are heterogeneously distributed in a CRR and the diffusion length of the defect is described by the size of the CRR. The average size of the CRR, $\xi$, at $T_{g}$ can be estimated from calorimetric data using the equation for the volume of CRR proposed by Donth [43]:

$$
\xi^{3} \equiv \mathrm{V}_{\alpha}=\frac{K_{B} T_{g}{ }^{2} \Delta\left(C_{v}^{-1}\right)}{\rho(\delta T)^{2}}
$$

where $\rho$ is the density, $\delta \mathrm{T}$ is the mean temperature fluctuation and $\mathrm{C}_{\mathrm{v}}$ is the isochoric heat capacity given by

$$
\Delta\left(\mathrm{C}_{\mathrm{v}}^{-1}\right)=\mathrm{C}_{\mathrm{vg}}^{-1}-\mathrm{C}_{\mathrm{vl}}^{-1}
$$

where $\mathrm{C}_{\mathrm{vg}}$ and $\mathrm{C}_{\mathrm{vl}}$ are respectively the isochoric heat capacities of the glass and the supercooled liquid extrapolated to $T_{g}$. Hempel et al.[44] derived the following relationship between $C_{v}$ and $C_{p}$ :

$$
\Delta\left(\mathrm{C}_{\mathrm{v}}^{-1}\right)=(0.74 \pm 0.22) * \Delta\left(\mathrm{C}_{\mathrm{p}}^{-1}\right)
$$

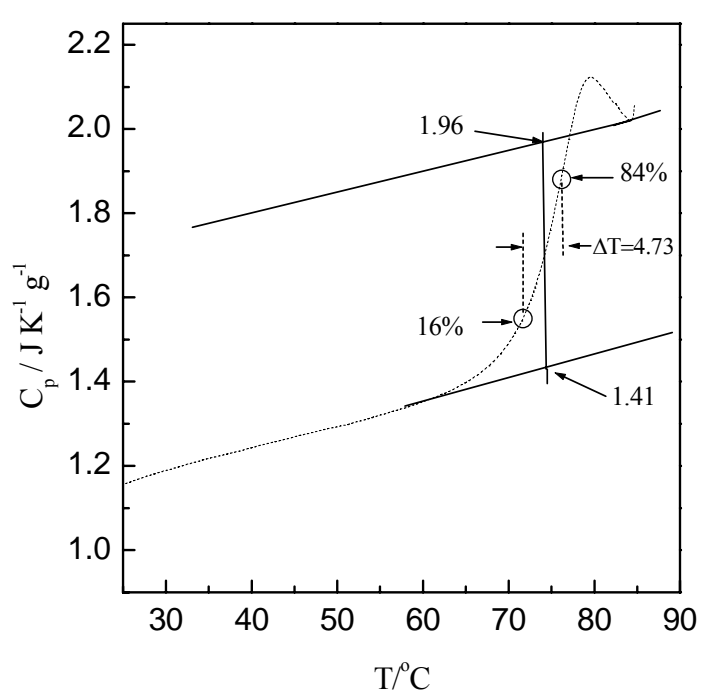

Fig. 6. Temperature dependence of the heat capacity for sucrose. The heat capacity at the glass transition (346 K) changes from 1.41 to

$1.96 \mathrm{~J} \mathrm{~K}^{-1} \mathrm{~g}^{-1}$. $\Delta \mathrm{T}$ is determined as the temperature interval in which $C_{p}$ changes from 16 to $84 \%$ of the total $\Delta C_{p}$.

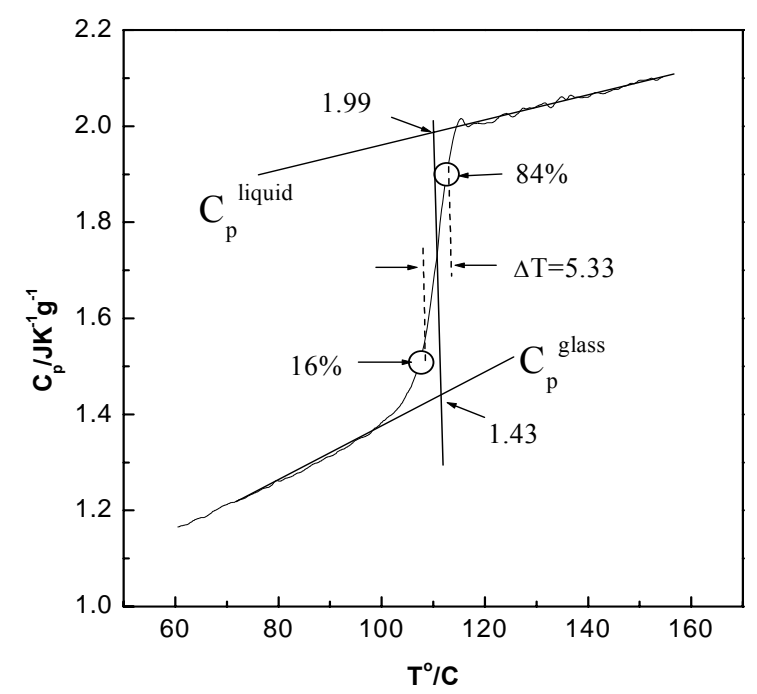

Fig. 7. Temperature dependence of the heat capacity for trehalose. The heat capacity at the glass transition $(390 \mathrm{~K})$ changes from 1.43 to $1.99 \mathrm{~J} \mathrm{~K}^{-1} \mathrm{~g}^{-1} . \Delta \mathrm{T}$ is determined as the temperature interval in which $C_{p}$ changes from 16 to $84 \%$ of the total $\Delta C_{p}$. Data are obtained the frequencydependence of $\mathrm{Tg}$ (TOPEM model)

$\delta \mathrm{T}$ is given by $(\Delta T / 2.5)$ [44] where $\Delta T$ is the temperature interval over which $\Delta C_{p}$ increases from 16 to $84 \%$ of the total $\Delta C_{p}$ at $\mathrm{T}_{\mathrm{g}}$. The estimation of $\Delta T$ for sucrose and trehalose are shown in Figures 6 and 7 respectively. The heat capacity data for both sucrose and trehalose are in excellent agreement with those reported by Shamblin et al.[13] Using equations 7-9, the CRR values were calculated to be $40.4 \mathrm{~nm}^{3}$ and $35.6 \mathrm{~nm}^{3}$ respectively for sucrose and trehalose. Assuming a spherical shape, the diameters of the CRR were calculated to be 3.4 and 3.3 nm respectively for sucrose and trehalose. These values are in agreement with the reported range of $2-5 \mathrm{~nm}$ for saccharides and polyalcohols [44].

Since the diameter of the CRR is a measure of the length scale of dynamic heterogeneity, it can be concluded that sucrose and trehalose are characterized by similar dynamic heterogeneity at $\mathrm{T}_{\mathrm{g}}$. The practical implications of these values for CRR could be explained following the argument of Vyazovkin et al.[22].

They found that the critical size of indomethacin nucleus was smaller than that of the CRR and thus it was argued that a critical nucleus could be formed even without involving cooperative motions. In other words, 
nucleation (and subsequently crystallization) could occur at temperatures where only local motions exist. We did not find in the literature the data necessary to calculate the critical size of sucrose and trehalose nuclei.

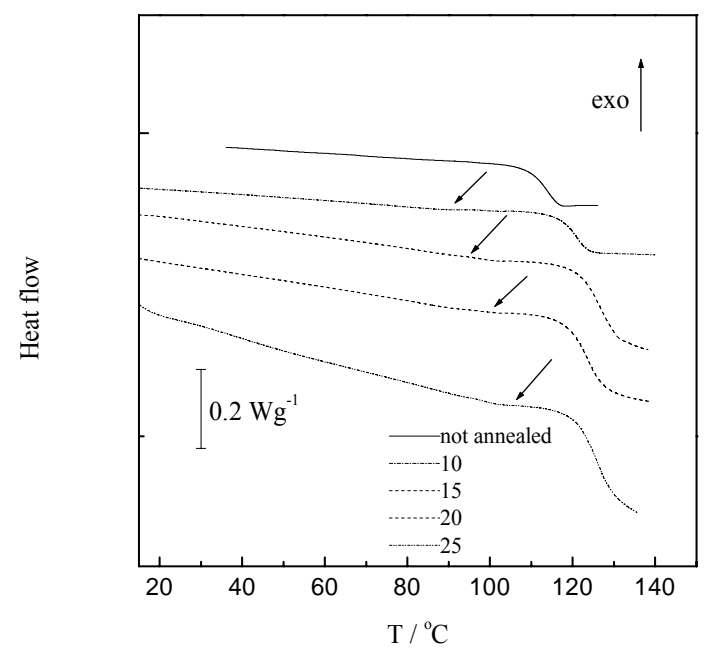

Fig. 8. DSC curves obtained on heating trehalose at different heating rates ranging from $10 \mathrm{to}^{2} 5^{\circ} \mathrm{C} \mathrm{min} \mathrm{m}^{-1}$ after annealing for $30 \mathrm{~min}$ at $60^{\circ} \mathrm{C}$. The heating rates $\left({ }^{\circ} \mathrm{C} \mathrm{min}^{-1}\right)$ are shown in the legend. "Not annealed" denotes a curve obtained without annealing at $10^{\circ} \mathrm{C} \mathrm{min}-1$ Arrows show the location of the shallow $\beta$-relaxation peaks.

But we could speculate that if the critical size of the sucrose and trehalose nuclei is smaller than that of the corresponding CRR, then nucleation could take place in these systems even in the absence of cooperative motions. Donth[43] also suggested an equation to calculate the number of molecules, $\mathrm{N}_{\alpha}$, constituting the CRR:

$$
N_{\alpha}=\frac{R T_{g} \Delta\left(C_{v}^{-1}\right)}{M(\delta T)^{2}}
$$

where $\mathrm{R}$ is the universal gas constant and $\mathrm{M}$ is the molecular weight ( 342.3 for sucrose and trehalose). $\mathrm{N}_{\alpha}$ was calculated to be 141 for trehalose and 129 for sucrose and again these values are close to those calculated for other structurally similar compounds [44, 45].

\section{Local mobility or secondary relaxations in sucrose and trehalose}

In trehalose, $\beta$-relaxation peaks were detected after annealing at temperatures $\geq 35^{\circ} \mathrm{C}$ (Fig. 8) while in sucrose it was $\geq 0^{\circ} \mathrm{C}$. The $T_{\mathrm{p}}$ values (Equation 1) were determined from the

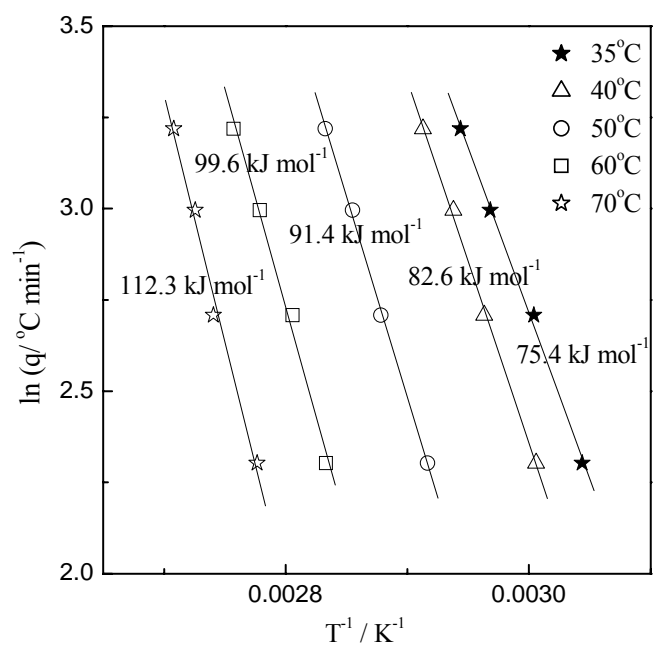

Fig. 9. Evaluation of activation energies from $\beta$-relaxation peaks of trehalose annealed at different temperatures (values in ${ }^{\circ} \mathrm{C}$ show the respective annealing temperatures). 
DSC curves after appropriate baseline subtraction. The annealing time was 30 minutes. As the annealing temperature increased, the $\beta$-relaxation peaks shifted towards the glass transition temperature. We did not use annealing temperatures above the respective upper limits $\left(10^{\circ} \mathrm{C}\right.$ for sucrose and $70^{\circ} \mathrm{C}$ for trehalose) to avoid any significant overlap of the $\beta$-relaxation peaks with the glass transition.

For each annealing temperature, the $\beta$-relaxation peak temperature expectedly increased with increase in the heating rate (Fig. 8). The plots $\ln \mathrm{q}$ vs $T^{-1}$ are shown in Figs. 9 and 10 for trehalose and sucrose respectively. The activation energy values calculated using Equation 1 are 42, 59, and $74 \mathrm{~kJ} \mathrm{~mol}^{-1}$ for sucrose (Figs. 9 and 11) whereas those for trehalose varied from 75 at an annealing temperature of $35^{\circ} \mathrm{C}$ to $112.3 \mathrm{~kJ} \mathrm{~mol}^{-1}$ at $70^{\circ} \mathrm{C}$ (Figs. 8 and 10 ). These values are much smaller than the activation energies of the $\alpha$-relaxation of sugars, typically in the $200-400 \mathrm{~kJ}$ $\mathrm{mol}^{-1}$ range.

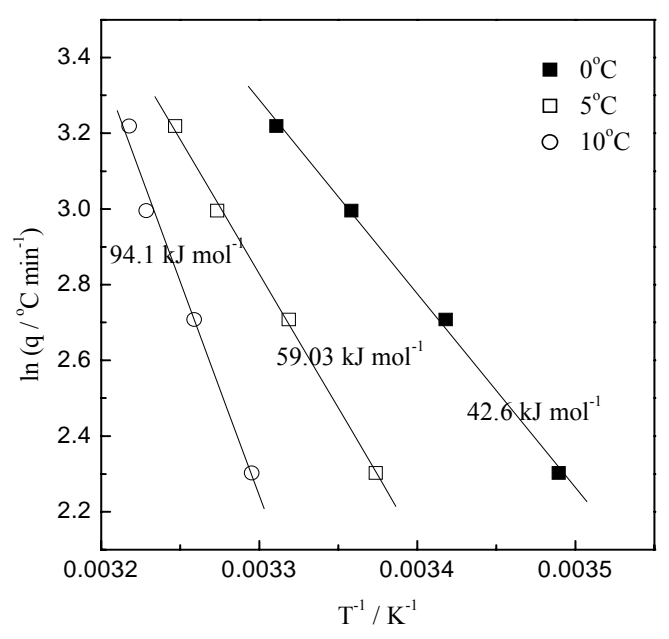

Fig.10. Evaluation of activation energies from $\beta$-relaxation peaks of sucrose annealed at different temperatures (values in ${ }^{\circ} \mathrm{C}$ show the respective annealing temperatures).

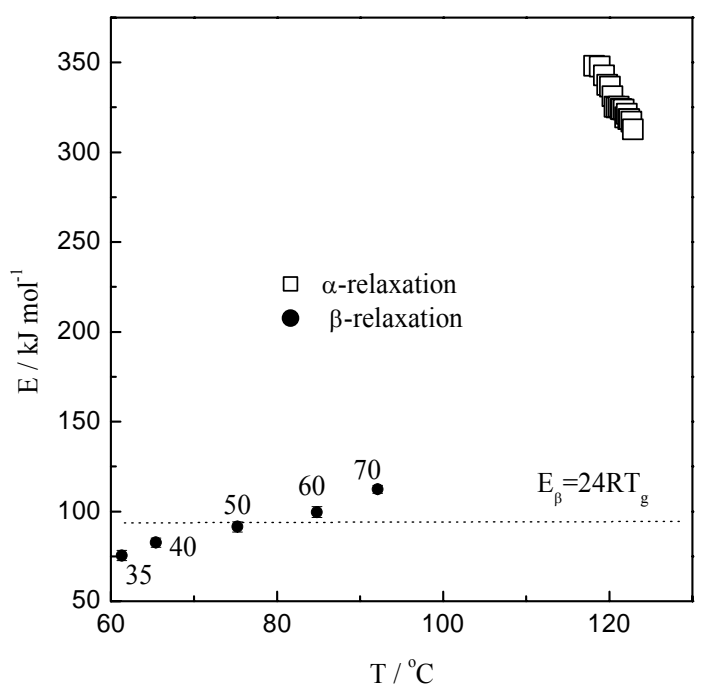

Fig. 11. Variation of the activation energies for the sub- $T_{g}$ (solid symbols) and $T_{g}$ (open symbols) events of trehalose with the average temperature of the process. Numbers by the points represent annealing temperatures. The dotted line corresponds to the value of the activation energy of $\beta$-relaxation calculated using Kudlik's empirical equation shown on the plot. Standard deviations of activation energies for $\beta$ relaxations are also shown.

The increase in activation energy with annealing temperature is due to the increasing contribution of the cooperative molecular motion that starts to unfreeze as the annealing temperature approaches $\mathrm{T}_{\mathrm{g}}$. Therefore, the activation energy value obtained at the lowest annealing temperature at which $\beta$-relaxation was detected is the best approximation of the activation energy of the "true" local molecular motions. 
Gusseme et al.[46] reported activation energy values of 47.2 and $85.6 \mathrm{~kJ} \mathrm{~mol}^{-1}$ for the two $\beta$-relaxations detected in amorphous trehalose. Of the two $\beta$-relaxations, the one with the activation energy of $85.6 \mathrm{~kJ} \mathrm{~mol}^{-1}$ occurs at temperatures close to those in our study. Since calorimetric methods yield activation energy values that are typically within $20 \%$ of those obtained from dielectric and/or mechanical spectroscopy[47], our value of $75 \mathrm{~kJ} \mathrm{~mol}^{-1}$ obtained at the lowest annealing temperature probably reflects the contribution from the $\beta$-relaxation with the higher activation energy. The contribution from some cooperative motion is also likely. For sucrose, our best estimate of the activation energy of $\beta$-relaxation is $42 \mathrm{~kJ} \mathrm{~mol}^{-1}$, a value in agreement that for other saccharides. The activation energy for $\beta$-relaxation was also calculated using the empirical equation, $E_{\beta}=(24 \pm 3) R T_{\mathrm{g}}$, derived by Kudlik et al.[48] The calculated and experimental values were in good agreement (Figs. 11 and 12).

It is instructive to evaluate the significance of the annealing temperatures at which $\beta$-relaxations were detected. The annealing temperatures ranged from $\left(T_{g}-73\right)$ to $\left(T_{g}-63\right)$ for amorphous sucrose $\left(T_{g}=73^{\circ} \mathrm{C}\right)$ and from $\left(T_{g}-84\right)$ to $\left(\mathrm{T}_{\mathrm{g}}-49\right)$ for amorphous trehalose $\left(\mathrm{T}_{\mathrm{g}}=119^{\circ} \mathrm{C}\right)$. As mentioned before, the activation energy increased with annealing temperature indicating an increase in the contribution from cooperative $\alpha$-relaxations. This observation is clearly in contrast to the generally held belief that mobility in amorphous pharmaceuticals is negligible at or below $\left(T_{g}-50\right)$.

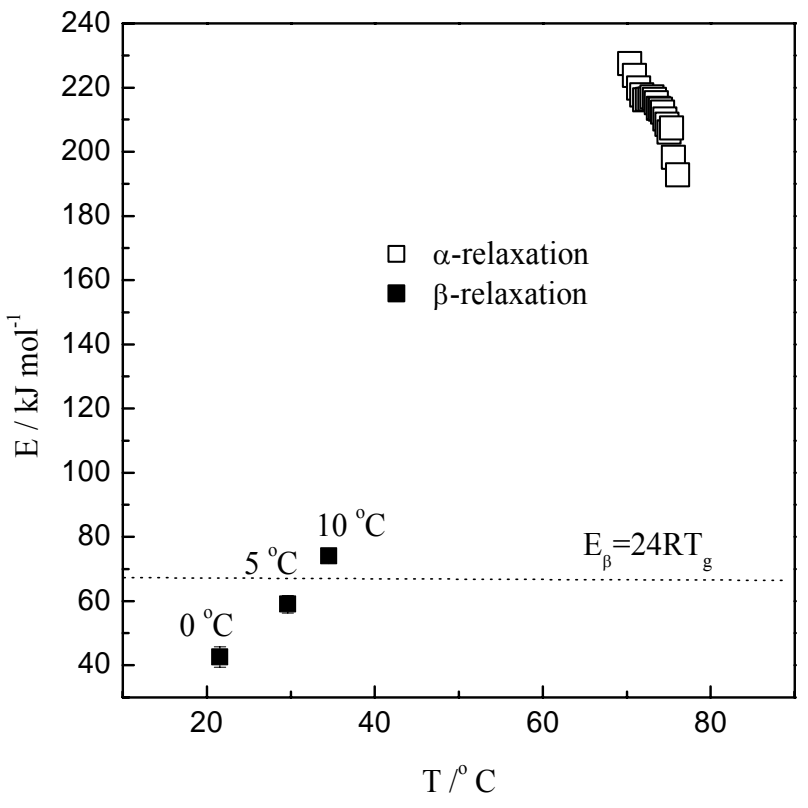

Fig.12. Variation of the activation energies for the sub- $T_{g}$ (solid symbols) and $T_{g}$ (open symbols) events of sucrose with the average temperature of the process. Numbers by the points represent annealing temperatures. The dotted line corresponds to the value of the activation energy of $\beta$-relaxation calculated using Kudlik's empirical equation shown on the plot. Standard deviations of activation energies for $\beta$ relaxations are also shown.

This becomes especially significant if we consider the fact that the annealing time in all the cases was only 30 minutes. It is clear that increasing the annealing time would result in an increase in the cooperative motions at the annealing temperatures used in our experiments $\left(<\mathrm{T}_{\mathrm{g}}-50\right)$. Since the average shelf-life of pharmaceuticals is about 2 years, the combination of such long storage (annealing) times and possibly higher storage (annealing) temperatures could result in sufficient mobility in the amorphous matrices of sucrose and trehalose to cause crystallization thereby potentially destabilizing the protein/peptide in the formulation.

The lowest annealing temperature (for the annealing time of 30 minutes) at which $\beta$-relaxation was detected for sucrose was much lower than that for trehalose and corresponds to the difference in the $T_{g}$ of these two compounds. The activation energy of $\beta$-relaxation at the lowest annealing temperature for sucrose $\left(\sim 42 \mathrm{~kJ} \mathrm{~mol}^{-1}\right)$ was also considerably lower than that for trehalose $\left(\sim 75 \mathrm{~kJ} \mathrm{~mol}^{-1}\right)$ suggesting greater ease in local motions in sucrose that could be very important if molecular mobility is coupled to stability. The increase in the activation energy of $\beta$-relaxation with annealing temperature in sucrose $\left(\sim 3.2 \mathrm{~kJ} \mathrm{~mol}^{-1} \mathrm{~K}^{-1}\right)$ is more than that for trehalose $\left(\sim 1.1 \mathrm{~kJ} \mathrm{~mol}^{-1} \mathrm{~K}^{-1}\right)$. This signifies that the effect of temperature on the increase in cooperative $\alpha$-relaxations is much more in sucrose than in trehalose, attributable to the lower free volume in amorphous trehalose. The implication of this is that even if these amorphous compounds are stored at temperatures $<\left(\mathrm{T}_{\mathrm{g}}-50\right)$, for the same increase in temperature, the increase in global mobility would be much more for amorphous sucrose. This could ultimately make the amorphous sucrose matrix more prone to instability compared to amorphous trehalose. This is counterintuitive and should not be confused with the fact that sucrose and trehalose, as supercooled liquids, have very similar dynamic fragility values. 
Our discussions above focus on the differences in the effects of temperature on the contributions from cooperative motions in the glassy state.

\section{Conclusions}

The local and global mobility, in two popular amorphous pharmaceutical excipients, sucrose and trehalose, have been compared. Trehalose, having a lower free volume in the glassy state due to a more tightly packed structure, experiences a greater effect of temperature on the reduction in the activation energy barrier for the glass to start flowing. This could be important in causing collapse of a lyophilized cake in that a small increase in temperature around the glass transition temperature of a formulation containing trehalose could cause pronounced viscous flow. In spite of a large difference in $\mathrm{T}_{\mathrm{g}}$, sucrose and trehalose were shown to have similar size of cooperatively rearranging regions (CRR) and also approximately the same number of molecules constituting the CRR, thus implying similar dynamic heterogeneity.

The minimum annealing temperature at which $\beta$-relaxation was detected was much lower in sucrose $\left(0^{\circ} \mathrm{C}\right)$ than in trehalose $\left(35^{\circ} \mathrm{C}\right)$ implying that progression of local motions to cooperative motions occurred at lower temperatures in sucrose. The increase in cooperative motions as a function of annealing temperature was much more for amorphous sucrose when stored below $\left(\mathrm{T}_{\mathrm{g}}-50\right)$. Thus even a small excursion in temperature could result in significant increase in mobility. This underscores the need to consider the mobility below the $\mathrm{T}_{\mathrm{g}}$ of an individual component or the effective $\mathrm{T}_{\mathrm{g}}$ of a multicomponent amorphous system.

\section{References}

[1]. Pouton, C. W. Formulation of poorly water-soluble drugs for oral administration: Physicochemical and physiological issues and the lipid formulation classification system. Eur. J. Pharm. Sci. 2006, 9:278-287. 2

[2]. Wang, W. Instability, stabilization and formulation of liquid protein pharmaceuticals. Int. J. Pharm. 1999, 185:129-188.

[3]. Yu L. Amorphous pharmaceutical solids: preparation, characterization and stabilization. Advanced Drug Delivery Reviews, 2001, 48:27-42.

[4]. Hancock, B. C.; Zografi G., Characteristics and significance of the amorphous state in pharmaceutical systems. J. Pharm Sci. 1997, 86:1-12.

[5]. Craig, D. Q. M.; Royall, P.G.; Kett, V. L.; Hopton, M. L.The relevance of the amorphous state to pharmaceutical dosage forms: glassy drugs and freeze-dried systems. Int. J. Pharm. 1999, 179:179-207.

[6]. Kreilgaard, L.; Frokjaer, S.; Flink, J. M.; Randolph, T.W.; Carpenter, J. F. Effects of additives on the stability of Humicola lanuginosa lipase during freeze-drying and storage in the dried solid. J Pharm Sci, 1999, 88:281-290.

[7]. Sarciaux, J. M. E.; Hageman, M. Effects of bovine somatotropin (rbSt) concentration at different moisture levels on the physical stability of sucrose in freeze-dried rbSt/sucrose mixtures, J. Pharm Sci , 1997, 86:365-371.

[8]. Izutsu, K.; Yoshioka, S.; Kojima, S. Physical stability and protein stability of freeze-dried cakes during storage at elevated temperatures, Pharm. Res. 1994, 11:995-999.

[9]. Zeng, X. M.; Martin, G. P.; Marriott, C. Effects of molecular weight of polyvinylpyrrolidone on the glass transition and crystallization of co-lyophilized sucrose. Int. J. Pharm., 2001, 218:63-73.

[10]. Passot, S.; Fonseca, F.; Alarcon-lorca, M.; Rolland, D.; Marin. M. Physical characterisation of formulations for the development of two stable freeze-dried proteins during both dried and liquid storage. Eur. J. Pharm. Biopharm. 2005, 60:335-348.

[11]. Liao, Y. H.; Brown, M. B.; Nazir, T.; Quader, A. ; Martin, G. P. Effects of sucrose and trehalose on the preservation of the native structure of spray-dried lysozyme. Pharm Res. 2002, 19:1847-1853.

[12]. Sun, W. Q.; Davidson, P. Protein inactivation in amorphous sucrose and trehalose matrixes: effects of phase separation and crystallization. Biochim Biophys Acta, 1998, 1425:235-244.

[13]. Shamblin, S. L.; Tang, X.; Chang, L.; Hancock, B.C.; Pikal, M. J. Characterization of the time scales of molecular motion in pharmaceutically important glasses. Journal of Physical Chemistry B, 1999, 103:4113-4121.

[14]. Hancock, B, C.; Shamblin, S. L. Molecular mobility of amorphous pharmaceuticals determined using differential scanning calorimetry. 2001, 380:95-107.

[15]. Hancock, B. C.; Shamblin, S. L.; Zografi, G. Molecular mobility of amorphous pharmaceutical solids below their glass transition temperatures. Pharm. Res. 1995, 12:799-806.

[16]. Weuts, I.; Kempen, D.; Six. K.; Peeters, J.; Verrec, G.; Brewster, M.; Van den Mooter, G. Evaluation of different calorimetric methods to determine the glass transition temperature and molecular mobility below $\mathrm{T}_{\mathrm{g}}$ for amorphous drugs.2003, Int. J. Pharm. 2003, 259:17-25.

[17]. Johari, G. Intrinsic mobility of molecular glasses. J Chem Phys, 1973, 58:1766 - 1770.

[18]. Johari, G. P.; Goldstein, M. Viscous liquids and the glass transition. II. Secondary relaxations in glasses of rigid molecules. J Chem Phys, 1970, 2372 - 2388.

[19]. Ngai, K. L. 2003. An extended coupling model description of the evolution of dynamics with time in supercooled liquids and ionic conductors. J Phys: Condens Matter. 2003, 15:S1107-S1125. 
[20]. Hikima, T.; Hanaya, M.; Oguni, M. Microscopic observation of a peculiar crystallization in the glass transition region and b-process as potentially controlling the growth rate in triphenylethylene. J. Mol. Struct., 1999, 479:245-250.

[21]. Alie, J.; Menegotto, J.; Cardon, P.; Duplaa, H.; Caron, A.; Lacabanne, C.; Bauer, M. 2004. Dielectric study of the molecular mobility and the isothermal crystallization kinetics of an amorphous pharmaceutical drug substance. J. Pharm. Sci., 2004 93:218 - 233.

[22]. Vyazovkin, S.; Dranca, I. Physical stability and relaxation of amorphous indomethacin. J. Phys. Chem. B, 2005, 109:18637-18644.

[23]. Vyazovkin, S.; Dranca, I. Probing beta relaxation in pharmaceutically relevant glasses by using DSC. Pharm. Res., 2006, 23:422-428.

[24]. Chen, H. S. On the mechanisms of structural relaxation in a $\mathrm{Pd}_{48} \mathrm{Ni}_{32} \mathrm{P}_{20}$ glass. J Non-Cryst Solids, 1981, 46:289-305.

[25]. V. A. Bershtein, V. M. Egorov. Differential Scanning Calorimetry of Polymers. ed., New York: Ellis Horwood, 1994.

[26]. Shamblin, S. L.; Taylor, L. S.; Zografi, G. Mixing behavior of colyophilized binary systems. J Pharm. Sci, 1998, 87(6):694-701.

[27]. Vyazovkin, S. Evaluation of the activation energy of thermally stimulated solid-state reactions under an arbitrary variation of the temperature. J. Comput. Chem., 1997, 18:393-402.

[28]. Vyazovkin, S. Modification of the integral isoconversional method to account for variation in the activation energy. J. Comput. Chem., 2001, 22:178-183.

[29]. Vyazovkin S.; Sbirazzouli, N. Isoconversional kinetic analysis of thermally stimulated processes in polymers. Macromolecular Rapid Communications, 2006, 27:1515-1532.

[30]. Ozawa, T. A new method of analyzing thermogravimetric data. Bull. Chem. Soc., Jpn., 1965, 38:1881-1886.

[31]. Flynn, H. J.; Wall, L. A. General treatment of the thermogravimetry of polymers. Journal of Research of the National Bureau of Standards, 1966, 70A:487-523.

[32]. Vyazovkin, S.; Sbirazzouli, N.; Dranca, I. Variation in activation energy of the glass transition for polymers of different dynamic fragility. Macromol. Chem. and Physics, 2006, 207:1126-1130.

[33]. Hancock, B. C.; Dalton, C. R.; Pikal, M. J.; Shamblin, S. L. A pragmatic test of a simple calorimetric method for determining the fragility of some amorphous pharmaceutical materials. Pharm Res., 1998, 15:762-767.

[34]. Green, J. E.; Sitaula, R.; Fowler, A.; Toner, M.; Bhowmick, S. Enthalpic relaxation of convective desiccated trehalose-water glasses. Thermochim Acta, 2007, 453:1-8.

[35]. Bhugra, C.; Rambhatla, S.; Bakri, A.; Duddu, S. P.; Miller, D. P.; Pikal, M. J,; Lechuga-Ballesteros, D. Prediction of the onset of crystallization of amorphous sucrose below the calorimetric glass transition temperature from correlations with mobility. J. Pharm. Sci., 2007, 96:1258-1269.

[36]. Phillips, J. C. Ideally glassy hydrogen-bonded networks. Phys Rev B: Condens Matter., 2006 73:024210-024211.

[37]. Wang, L. M.; Velikov, V.; Angell, C. A. Direct determination of kinetic fragility indices of glassforming liquids by differential scanning calorimetry: Kinetic versus thermodynamic fragilities. J Chem Phys., 2002, 117:10184-10192.

[38]. Wang, L. M.; Angell, C. A. Response to "Comment on 'Direct determination of the fragility indices of glassforming liquids by differential scanning calorimetry: Kinetic versus thermodynamic fragilities' " [J. Chem. Phys. 118, 10351 .2003]. J Chem Phys 118:10353-10355.

[39]. Wang, L. M.; Angell, C, A.; Richert, R. 2006. Fragility and thermodynamics in nonpolymeric glass-forming liquids. J. Chem. Phys., 2006, 125:074505-074501-074508.

[40]. Higashiyama, T. Novel functions and applications of trehalose. Pure Appl Chem., 2002, 74:1263-1269.

[41]. Adam, G.; Gibbs, J. H., On the temperature dependence of cooperative relaxation properties in glass-forming liquids. J. Chem. Phys, 1965, 43:139-146.

[42]. Glarum, S. H. Dielectric relaxation of isoamyl bromide. J. Chem. Phys, 1960, 33:639-643.

[43]. Donth E. The Glass Transition: Relaxation Dynamics in Liquids and Disordered Materials. ed., Berlin: Springer 2001

[44]. Hempel, E.; Hempel, G.; Hensel, A.; Schick, C.; Donth, E. Characteristic length of dynamic glass transition near $\mathrm{T}_{\mathrm{g}}$ for a wide assortment of glass-forming substances. J. Phys. Chem. B, 2000, 104:2460-2466.

[45]. Vyazovkin , S.; Dranca, I. Comparative relaxation dynamics of glucose and maltitol. Pharm Res., 2006, 23:2158-2164.

[46]. Gusseme, A. D.; Carpentier, L.; Willart, J. F.; Descamps, M. Molecular Mobility in supercooled trehalose. J. Phys. Chem. B, 2003, 107:10879-10886.

[47]. Vyazovkin, S.; Dranca I. Activation energies derived from the pre-glass transition annealing peaks. Thermochim Acta, 2006, 446:140-146.

[48]. Kudlik, A.; Benkhof, S.; Blochowicz, T.; Tschirwitz, C.; Rossler, E. The dielectric response of simple organic glass formers. J Mol Struct., 1999, 479:201-218. 